\title{
Chaperon Co-chaperon interaction modulators prevents cancer and neurodegenerative diseases.
}

\author{
Previn R * \\ Department of Dental and Neuroscience University of Central Lancashire, UK
}

\section{Editorial}

The Heat shock proteins (HSPs) are molecular chaperons which regulates protein homeostasis. Any changes in this process can cause different diseases like neurodegenerative diseases, and cancer. The widely studied and used critical chaperons are Hsp 70 and Hsp 90 [1]. The heat shock factor-1 (HSF-1) is a transcriptional activator for Hsp70 chaperon protein. In normal condition, HSF-1 maintains its inactive (monomeric) form by binding to Hsp90 protein. But in dysregulated intracellular homeostatic conditions HSF-1 separates from Hsp90 and transfers for upregulation of Hsp genes. The HSF-1 has ability to trigger the Hsp70 expression. Currently, Celastrol and Carbenoxolone drugs are available as a HSF-1 modulator. The pharmacological status of these drugs includes short-term clinical trials for rheumatoid arthritis and phase II clinical trials for psoriasis. But it has limitations like strong human toxicity. The Hsp90 inhibitors have ability to inhibit the interaction between Hsp90 and HSF-1, which is important for Hsp70 expression and activity. Commonly used Hsp90 inhibitors are Geldanamycin, 17-AAG (17-N-allylamino-17-demethoxygeldanamycin), 17- DMAG (17-Dimethylaminoethylamino-17-demethoxygeldanamycin), SNX-2112 used in clinical trials for the cancer but discontinued due to its toxic effect and poor blood brain barrier penetration. The intracellular protein homeostasis is maintained by chaperon protin regulation. The Hsp110, Hsp 40, NEF (BAG) and TPR (Hip) co-chaperons has critical role of ATP hydrolysis and client selection for Hsp70 (14). In further processes, Hsp40 (NEF)/ Hip (TPR) instructs Hsp70 for client stability on the other hand BAG (NEF)/ HSP110 are the destabilizes the interaction. This data directly suggest that the co-chaperons has major role in Hsp70 function. The important co-chaperons involved in this process are J proteins, tetratricopeptide repeat (TPR) domain, nucleotide exchange factors (NEF) [1]. Previous studies has discussed about the Hsp70 structure which contain SBD and $\mathrm{NBD}$, the role of co- chaperons like J-domain on substrate and Hsp70 resulting in ATPhydrolysis at NBD allowing SBD to capture substrate of Hsp70, NEF role in making Hsp70 available for substrate binding in next cycle endorsing polypeptide unfolding -refolding localized events, Efficiency of TPR for HSP 70 function $(1,2,11,12)$. More attention is required for the study of these chaperon co-chaperon protein interactions (CCI) which can be a new therapeutic target in different disease models. The major function of this interaction (CCI) is protein folding, trafficking and degradation of protein aggregation. In neurodegeneration diseases HSP70 activation is important this can be achieve by using a molecules with promising protagonist of HSP 70 activation for protein disaggregation [2]. Currently many stimulators and inhibitors of chaperon available but they showed limitations for its use. HSP90 inhibitor treatment for longer period may cause degradation and disruption of its function [2]. On the opposite side HSP 70 overexpression triggers tumor progression and development by stabilizing oncogenic kinases. The HSP70 binds to a specific region on co-chaperon for its function and the discovery of small molecules with the ability to block or modulate this interaction in mutated or dysregulated Hsps can play major role in the treatment therapy for different diseases. The small molecules play role as an inhibitor for chaperon co-chaperon interaction (CCI) by targeting the binding site for Hsp70 on co-chaperon and to blocks its expression. These inhibitors provide the novel therapeutic approach in cancer [3]. Researchers should focus on the interactions between Hsp70 and its co-chaperones as a new therapeutic target. It is also important to study CCI in detail and the mechanism involved in action of activator/inhibitor on CCI. The treatment therapy with low toxicity CCI stimulating/ activating molecule of Hsp 90 can be the effective therapy in Cancer disease.

The difficulty in this approach is to target CCIs (Hsps and cochaperon). Development of Novel therapies is growing research topic in this area. The Hsps might be an attractive target for developing high number of CCI in guiding Hsps for the interaction.

\section{References}

1. Kalia KS, Kalia VL, McLean JP. Molecular chaperones as rational drug targets for Parkinson's disease therapeutics. CNS Neural Disord Drug Targets. 2010; 9(6): 741-753.

2. Reis SD, Pinho BR, Oliveira JM. Modulation of molecular chaperons in Huntington's disease and other polyglutamine disorders. Molecular Neurobiology. 2016; 12(4): 345-357.

3. Ciocca DR, Calderwood SK. Heat shock proteins in cancer: Diagnostic, prognostic, predictive, and treatment implications. Cell Stress \& Chaperones. 2005; 15(3): 256-268.

\section{*Correspondence to:}

R Previn

Department of Dental and Neuroscience, University of Central Lancashire,

UK

E-mail: r.previn2903@yahoo.com 